\title{
Globe
}

Revue internationale d'études québécoises

\section{Réal La Rochelle : L'opéra du samedi. Le Metropolitan à la radio du Québec, Québec, Les Presses de l'Université Laval, 2008}

\section{Marie-Thérèse Lefebvre}

Volume 12, numéro 2, 2009

URI : https://id.erudit.org/iderudit/1000718ar

DOI : https://doi.org/10.7202/1000718ar

Aller au sommaire du numéro

Éditeur(s)

Globe, Revue internationale d'études québécoises

ISSN

1481-5869 (imprimé)

1923-8231 (numérique)

Découvrir la revue

Citer ce compte rendu

Lefebvre, M.-T. (2009). Compte rendu de [Réal La Rochelle : L'opéra du samedi. Le Metropolitan à la radio du Québec, Québec, Les Presses de l'Université Laval, 2008]. Globe, 12(2), 211-215. https://doi.org/10.7202/1000718ar d'utilisation que vous pouvez consulter en ligne.

https://apropos.erudit.org/fr/usagers/politique-dutilisation/ 
mais ils offrent un éventail d'arguments qui alimentent certainement la réflexion, même s'ils n'apportent rien de bien neuf au débat. Puisque les nouveaux programmes sont implantés, il faudrait peut-être cesser de discuter des enjeux politiques ou pédagogiques que leur création a soulevés, et les laisser subir l'épreuve du réel avant de procéder à un véritable bilan de leurs succès et de leurs ratés. Il sera alors temps, et peut-être nécessaire, de réformer le "renouveau pédagogique".

Dominique Marquis

Université du Québec à Montréal

\section{Réal La Rochelle, L'opéra du samedi. Le Metropolitan à la radio du Québec Québec, Les Presses de l'Université Laval, 2008.}

Si l'histoire de la radio au Québec est relativement bien connue aujourd'hui, l'analyse d'émissions spécifiques nous est beaucoup moins familière. Les travaux de Pierre Pagé et de Renée Legris ont ouvert la voie à ces recherches, particulièrement dans le domaine du théâtre radiophonique. Les séries Radio-Collège, 1941-1956 sont parmi les premières études sur les contenus radiophoniques. La publication de Réal La Rochelle vient donc à point combler une lacune en proposant un examen d'une des plus anciennes émissions toujours en ondes, L'opéra du samedi, dont la diffusion a débuté en 1931.

Né dans la région de l'Abitibi en 1937, l'auteur est un passionné d'opéra et de cinéma. Il est membre associé au Centre de recherche sur l'intermédialité (CRI) à l'Université de Montréal et président fondateur de la Phonothèque québécoise. Il a publié de nombreux essais dans le domaine de l'audiovisuel. Comme il le précise dans son introduction, ce livre n'est ni un ouvrage musicologique, ni un essai historique, ni une étude du répertoire ou

$$
+4
$$

1. Voir Marie-Thérèse LEFEBVRE, “Radio-Collège (1941-1956): un incubateur de la Révolution tranquillen, Les Cahiers des Dix, n60, 2006, p. 233-275; Kim PETIT, "Le projet pédagogique de RadioCollège dans la décennie 1940 : la conservation des institutions scolaires traditionnelles et la promotion des sciences", mémoire de maîtrise, Département d'hiscoire, Université de Sherbrooke, 2006; Dominic SPENCE, "Analyse de la série radiophonique "Musique de notre siècle" animée par le compositeur Serge Garant (1969 à 1985) ", mémoire de maîtrise, Département de musicologie, Universicé de Montréal, 2004. 
des interprètes. L'analyse qu'il propose cherche plutôt à saisir l'impact socioculturel de cette diffusion d'opéras du Metropolitan de New York sur les auditeurs francophones du Québec (p. 9). S'appuyant surtout sur les souvenirs des passionnés de cette émission, l'auteur, qui s'adresse à la fois aux amateurs d'opéras et aux historiens de la culture québécoise, atteint-il son objectif premier? Pour répondre à cette question, je devrai porter deux chapeaux : celui de la fidèle auditrice que j'ai été durant plusieurs années, et celui de la musicologue qui s'interroge sur la pertinence et la présentation de ce travail.

Pour les auditeurs de la génération des soixante ans et plus, principaux témoins sur lesquels s'appuie l'auteur tout au long de son propos et auxquels il s'adresse d'abord et avant tout, ce volume est un véritable feu d'artifice d'informations. Les étincelles éclatent dans tous les sens à partir d'une trame chronologique des faits. La Rochelle a en effet construit le récit comme une conversation à bâtons rompus entre des familiers de l'émission qui partagent leurs souvenirs.

Il rebondit sur l'anecdote de Laurent Major qui lui fournit le "scoop" de l'affaire Texaco, commanditaire de l'émission jusqu'en 2004 (le nom y est mentionné 22 fois).

Il réagit en trois pages (p. 66-69) au commentaire d'Edgar Fruitier (p. 136) sur "l'extase» de Saint-Denys Garneau envers la chanteuse Elizabeth Rethberg que ce dernier évoque en janvier et mars 1934 dans ses lettres à Jean Le Moyne, et l'auteur ajoute qu'il s'explique mal comment ce poète peut préférer l'opéra allemand à l'opéra italien, à ses yeux "fille de joie" ou simple divertissement, alors qu'il aime bien raconter ses ballades en voiture «avec les guidounes"! J'avoue que je m'explique mal le lien de cause à effet que tente de faire valoir l'auteur ici. La relation profonde de Saint-Denys Garneau à la musique peut-elle tenir en cette unique comparaison?

L'auteur rappelle aussi, au grand plaisir des amateurs, les réalisateurs et les animateurs qui ont occupé, d'hier à aujourd'hui, l'antenne du samedi, les interprètes légendaires du Met (la plupart ayant œuvré avant 1960), oubliant tout de même de signaler qu'au-delà de ces quelques auditions radiophoniques fabuleuses, nous avons aussi eu droit, durant toutes ces décennies, à un nombre incalculable de voix plus ou moins médiocres.

Il n'hésite pas cependant à critiquer les choix ultra-conservateurs des administrateurs du Met qui ont diffusé quelques opéras fort ennuyeux de compositeurs américains et qui ont présenté de manière routinière les opéras les plus populaires. Les diffusions radiophoniques du Met: un lieu de décou- 
vertes? Pelléas? Wozzeck? Non pas, mais personne, parmi les invités que l'auteur a choisi d'interroger, ne semble s'en plaindre. Tous rappellent que c'est là qu'ils ont découvert "la belle musique" (quelle serait leur définition de son opposé, "la musique laide» ?) et que ces auditions leur ont permis de construire leurs propres mises en scène imaginaires.

Les auditions du Met c'est aussi le lieu des batailles épiques que $\mathrm{La}$ Rochelle raconte avec plaisir. Callas contre Milanov, "qu'elle envoie à la guillotine" (p. 110), et contre Tebaldi, "qu'elle pourchasse avec les mêmes armes" (p. 110); Jobin contre Pavarotti pour le nombre de contre-ré, 2 contre 5 et peut-être même 10, dans La Fille du Régiment (la partition indique 9 contre-ut); mais aussi celle des trois "Jeanne d'Arc" qui ont sauvé l'institution à différentes périodes, Lucrezia Bori (1933), Lily Pons (1940) et Beverly Sills (2004). Comparer le nombre de spectateurs à ceux d'un combat de boxe (p. 222) pour une représentation de Carmen le 12 décembre 1952 n'étonne donc pas dans ce contexte.

Construit à la manière des innombrables discussions diffusées durant les entractes, ce livre plaira donc aux amateurs plus âgés qui se rappelleront ainsi "le bon vieux temps". Mais ce livre pourra-t-il susciter autant d'intérêt auprès des musicologues et des étudiants en musique, particulièrement des nombreux chanteurs en herbe inscrits dans les universités et conservatoires qui rêvent d'atteindre les hauts lieux du Met?

Il y a néanmoins de belles surprises dans le travail de La Rochelle. Plusieurs réflexions pourront être prolongées dans le cadre d'études portant sur l'histoire culturelle et musicale du Québec. On y découvre l'importance des critiques radiophoniques de Thomas Archer, critique musical au journal The Gazette de 1930 à 1970, et qui a, selon l'auteur, "élaboré une véritable techno-esthétique de la radio musicale d'opéra» (p. 47). Les quelques lignes qu'il lui consacre ouvrent la voie à un immense chantier de recherche encore presque vierge: celui d'une histoire de la critique musicale au Québec.

La contribution de la radio et du cinéma à la démocratisation de l'opéra (p. 81) à partir des années 1940 est un autre sujet de discussion intéressant pour le musicologue. Oui, le public pouvait entendre ces fameux airs d'opéras sans avoir à se déplacer, mais on lui offrait par contre très peu d'œuvres nouvelles à découvrir. Et c'est justement ce que déplorait SaintDenys Garneau.

Tous ces airs italiens, français et même allemands, étaient déjà archiconnus au Québec depuis la fin du dix-neuvième siècle alors qu'ils circulaient dans les foyers via les innombrables musiques en feuille en provenance d'Europe et des États-Unis. Des arrangements pour fanfare et 
harmonies étaient diffusés dans les lieux publics et, à partir de 1922, les hôtels de Montréal diffusaient tous les soirs des orchestrations de ce répertoire pour des émissions de musique "en dînant " ou "en dansant ". Ce n'est qu'au milieu des années 1930 que ce répertoire (populaire) d'opéra diffusé à la radio sera abandonné au profit de la chanson française. Et c'est sans parler des nombreuses troupes d'opéra étrangères et locales qui présentèrent ce même corpus, et ce, dès 1840 , ce que révèle la thèse de l'historienne Mireille Barrière'.

Le chant attirait de nombreux étudiants dans les cours privés des Rodolphe Plamondon, Salvator Issaurel, Arthur Laurendeau, Roger Filiatrault, Édouard J. Woolley, Albert Cornellier, Céline Marier, Jeanne Maubourg, Sarah Fischer, Pauline Donalda, pour ne nommer que ceux et celles qui étaient connus au moment où Joseph Rouleau débute ses études en 1947 avec Woolley et Cornellier. Il est vrai que le Conservatoire de musique du Québec à Montréal avait ouvert ses portes en 1943 et que la classe de chant ne sera formée qu'en 1951 avec la venue de Martial Singher. Mais Rouleau a quand même réussi à se faire connaître grâce à de nombreux concours, aux tournées organisées par les Jeunesses musicales du Canada que venait de fonder Gilles Lefebvre (1949), et aux rôles de soutien qu'il obtint à l'Opéra national du Québec et à l'Opéra Minute de Montréal. Le milieu l'avait donc préparé à acquérir une formation supérieure en Europe, grâce à une bourse du gouvernement. Le Québec était-il vraiment aussi «désert" que La Rochelle semble le dire (p. 151) ?

L'analyse qu'il présente des années 1950 où, écrit-il, les collèges classiques "moribonds» (p. 186) interdisaient, durant les huit ans que duraient les études, l'écoute de la radio (p. 121) me semble vouloir réduire cette période à une "grande noirceur" (p. 214), qualificatif qui n'a plus cours depuis que des historiens d'une nouvelle génération ont renouvelé l'interprétation de cette période qui annonce les grands bouleversements de la décennie suivante.

Il se peut que certains collèges en région aient été plus rigides et je ne mets pas en doute les souvenirs de La Rochelle et de Claude Routhier, mais, pour avoir fréquenté comme "pensionnaire" ces mêmes institutions dans les mêmes années 1950, je puis affirmer qu'au Collège Basile-Moreau (et il en était de même aux collèges Saint-Laurent, Brébeuf et Sainte-Marie),

$$
+4
$$

2. Mireille BARRIERE, "La société canadienne-française et le thêâtre lyrique à Montréal entre 1840 et 1913 ", thèse de doctorat, Département d'histoire, Université Laval, 1990. 
nous pouvions écouter le théâtre de Radio-College et l'Opéra du samedi si nous le souhaitions, et les étudiants avaient fondé des clubs du disque où j'ai été initiée au répertoire lyrique. Loin d'être moribonds, ces collèges ont formé la plupart des initiateurs de ladite Révolution tranquille. S’ils ont été abolis en 1964, c'est qu'un nombre beaucoup trop restreint d'étudiants y avaient accès. Ici encore, les propos de l'auteur appellent une étude plus approfondie de la place de la musique et des activités culturelles dans ces lieux privilégiés d'enseignement.

Pour répondre à la question de l'avenir de cette émission (et même de la radio) soulevée en épilogue (p. 211 et suivantes), l'auteur cède la parole à Georges Nicholson qui révèle que «lors de la première saison d'Espace Musique, 2004-2005, les cotes d'écoute de la station ont augmenté de $44 \%$ " (p. 227). Mais $44 \%$ de quel pourcentage d'auditeurs à la chaîne $100.7 ? 2 \%, 3 \%$ ?

Ce réquisitoire contre la création de la nouvelle structure de Radio-Canada établie il y a cinq ans est, hélas, déjà dépassé. Car il existe maintenant une nouvelle génération d'auditeurs et d'auditrices qui n'ont aucune référence à l'ancienne chaîne culturelle. Dans ces conditions, l'émission l'Opéra du samedi peut-elle espérer renouveler son auditoire?

Depuis une dizaine d'années, je pose la question "Quelles sont vos émissions radiophoniques préférées? " à des étudiants inscrits en musique dans un cadre universitaire. $47 \%$ d'entre eux répondent qu'ils n'écoutent jamais ou rarement la radio. Quant aux chanteurs de ces classes, aucun n'a dit connaître cette émission si chère à Réal La Rochelle. De quoi faire réfléchir...

Un mot, enfin, sur la présentation des notes et de la bibliographie, outils qui intéressent davantage les chercheurs. L'auteur et l'éditeur ont fait preuve de peu de discipline. On découvre ici et là des erreurs qui auraient pu être facilement évitées (par exemple, l'article de Claude Routhier dans la revue Aria auquel renvoie l'auteur [p. 186 et p. 253] date de 1980 et non de 1990), et l'organisation des notes démontre une absence totale de rigueur méthodologique. L'amateur de cette émission excusera cependant plus facilement ces lacunes car il découvrira, à son grand bonheur, des références fort pertinentes aux enregistrements historiques du corpus qu'évoque l'auteur tout au long de son essai et dont plusieurs sont maintenant accessibles sur Internet.

Marie-Thérèse Lefebvre

Université de Montréal 